\title{
Per 100 White Blood Cells
}

National Cancer Institute

\section{Source}

National Cancer Institute. Per 100 White Blood Cells. NCI Thesaurus. Code C67219.

Natural number unit for measurement of portion of a particular type of blood cells (such as any type of white blood cells or nucleated red blood cells) in the total white blood cell count expressed per count of 100. 